\title{
Grid preparation and data Collection for cryo-EM
}

\author{
Leifu Chang
}

Department of Biological Sciences, Purdue University, 915 W. State Street, West Lafayette, IN 47907. Email: Ichang18@purdue.edu

Cryo-EM has transformed structural biology and many other biological research areas due to recent technical advances including improved electron detectors and image-processing algorithms. Compared to X-ray crystallography, cryo-EM allows structural determination of large and heterogenous macromolecular complexes in their native state with very little sample. It is now possible, in some ideal cases, to determine a protein structure by single-particle cryo-EM from data collected in a few hours. Despite the advances, many challenges remain for routinely obtaining high-resolution structures. One of the challenges is grid preparation, which is usually decisive for successful data collection and structure determination. In my lectures. I will discuss how to design experiments for freezing cryo-EM grids and strategies for data collection. 